\title{
Congenital cytomegalovirus infections in Poland - a national hospital register-based study
}

\author{
Krzysztof Kanecki ${ }^{1, A, C-F \oplus}$, Aneta Nitsch-Osuch ${ }^{1, A, E-F} \oplus$, Paweł Goryński ${ }^{2, A-B, E-F} \oplus$,

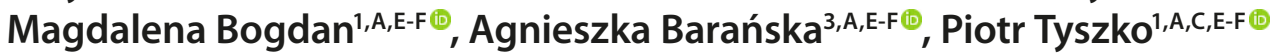 \\ ${ }^{1}$ Department of Social Medicine and Public Health, Medical University, Warsaw, Poland \\ ${ }^{2}$ National Institute of Public Health/National Institute of Hygiene, Warsaw, Poland \\ ${ }^{3}$ Department of Medical Informatics and Statistics with E-learning Laboratory, Medical University, Lublin, Poland \\ A - Research concept and design, B - Collection and/or assembly of data, C - Data analysis and interpretation, \\ $D$ - Writing the article, $E$ - Critical revision of the article, $F$ - Final approval of article
}

Kanecki K, Nitsch-Osuch A, Goryński P, Bogdan M, Barańska A, Tyszko P. Congenital cytomegalovirus infections in Poland - a national hospital register-based study. Ann Agric Environ Med. 2020; 27(4): 574-578. doi: 10.26444/aaem/128635

\section{Abstract}

Introduction and objective. Congenital cytomegalovirus (cCMV) infections are common worldwide. CCMV infected newborns are mostly asymptomatic at birth. However, cCMV remains the major infectious cause of congenital abnormalities in the developing foetus and newborns. The aim of the study was to provide recent data on cCMV in Poland.

Materials and method. A retrospective, population-based study was conducted using data from hospital discharge records of patients hospitalized from 2009-2018. CCMV incidence rates were estimated on the basis of data taken from a Polish hospital morbidity study, carried out by the National Institute of Public Health.

Results. The study group consisted of 1,916 hospitalized patients with the first-time diagnosis of cCMV. Among them, $52 \%$ were patients up to 30 days of age. The median age was 28 days. The average annual incidence of cCMV was 48 per $100,000$ live births (95\% $\mathrm{Cl} 23.5-73.4)$. A significantly higher CCMV incidence rate was observed in patients from more urban than rural regions of Poland ( 55 per 100,000 vs 39 per 100,$000 ; P<0.001)$. In the study period, 9 hospital deaths $(0.5 \%$ of all patients) were reported. The coexistence of nervous system diseases was observed in $26 \%$ of cases and hearing loss in $6 \%$ of cases.

Conclusions. The significantly more cCMV cases were observed in patients from more urban than rural regions of Poland may suggest the presence of territorial factors related to the CCMV incidence in Poland. The relatively high percentage of newborns with hearing loss or neurologic diseases may require activities aimed at reducing the cCMV incidence. The epidemiological data presented may be useful for comparisons with other geographical regions.

\section{Key words}

epidemiology, incidence, CMV, nationwide register

\section{INTRODUCTION}

Congenital cytomegalovirus (cCMV) infections are common worldwide. In developed countries, congenital cytomegalovirus is the most common infectious cause of malformations in newborns [1]. About $10 \%$ of infected newborns are symptomatic at birth, and nearly half of them are exposed to long-term sequelae. Hearing loss is claimed to be the most common long-term sequela $[2,3]$. What is more, infections caused by the human cytomegalovirus are one of the most common viral infections that are acquired by the human foetus and they may lead to central nervous system damage [4]. It was reported that the rate of materno foetal transmission is linearly correlated to the gestational age at infection [5]. Children of mothers with non-primary infections tend to be more symptomatic at birth than those of mothers with primary infections [6].

It can be observed in Europe that the first-time occurrence of CMV takes place in some women during pregnancy and that certain women are re-infected with a different virus

Address for correspondence: Krzysztof Kanecki, Department of Social Medicine and Public Health, Medical University of Warsaw, Oczki 3, 02-007, Warsaw, Poland E-mail:kanecki@mp.pl

Received: 18.08.2020; accepted: 20.10.2020; first published: 09.12.2020 strain. The incidence of transplacental transmission is estimated at $30 \%$ in cases related to primary infections and $2 \%$ in cases related to non-primary infections. The prospective risk of infection in seronegative pregnant women has been observed to be four times higher than the risk to which immune women are exposed [7]. Children of women who were seronegative during their first pregnancy and become pregnant again within the next two years are at the highest risk of developing cCMV-related sequelae [8]. A paper from the USA indicated that cCMV caused more developmental delays and long-term sequelae than Down syndrome (trisomy 21), neural tube defects, or oetal alcohol syndrome combined [9]. A systematic review showed that the cCMV prevalence in developed countries was at the level of $0.58 \%$ (95\% CI: $0.41-0.79$ ) [10]. In a five-year study conducted in the USA in a group of infants $<1$ year of age, the annual average of 747 cCMV-related hospitalizations was estimated (18.6/100,000 per year), 408 of which (55\%) occurred in infants $<1$ month of age (122.0/100,000 per year) [11]. In a selected population of American Indian and Alaska Native infants during 2000$2017,54(1.5 / 10,000)$ of 354,923 cases had a congenital CMVcoded diagnosis [12]. The cCMV incidence rates were likely to be higher in low- and middle-income countries [2, 13]. In a prospective study from Indonesia conducted at a national 
referral hospital in 2016-2017, cCMV infection was diagnosed in 411 newborns, which constituted $5.8 \%$ of the neonates subjected to the study [14]. In China, the overall prevalence of newborns diagnosed with the cCMV was reported to be 0.7\% (95\% CI: 0.5\%-0.9\%) [15]. A retrospective study from central Germany showed the prevalence of clinically relevant cCMV infections at the level $0.008-0.04 \%$. In addition, the risk of all cCMV infections was reported as underestimated by the authors, The true prevalence of clinically relevant and subclinical cCMV infections was reported to be $>0.04 \%$ [16]. A study from two European countries that focused on CMV screening in newborns in 1977-1986 estimated the incidence of congenital infections in infants at the level of 4.6 cases per 1,000 births in Malmö, and 3.2 cases per 1,000 births in London [17]. Information on CCMV in Poland is limited or based on case reports $[18,19,20,21,22]$. Due to its clinical and public health importance, recent data on cCMV epidemiology in Poland should be investigated.

\section{OBJECTIVE}

The aim of the study was to provide recent data on cCMV based on hospitalization data from national registers in Poland.

\section{MATERIALS AND METHOD}

In a retrospective, population-based study, the hospital discharge records of patients with cCMV diagnosis were analyzed. Data were obtained from the National Institute of Public Health in Poland and covered the period 2009-2018. All hospitals in Poland, with the exception of psychiatric facilities, are legally required to send discharge data to the Institute. The data are anonymous and include information on hospitalizations with ICD10-code diagnoses, dates of admission and discharge, date of birth, gender, and place of residence. In addition, demographic data for the general Polish population were obtained from Statistics Poland [23]. The criteria for dividing the study group into inhabitants of rural or urban areas were adopted in accordance with the methodology used by the National Institute of Public Health/ National Institute of Hygiene in Warsaw. These criteria relate to the assignment of patients based on TERYT residence codes.

Symptomatic cCMV patients often require hospitalization, and therefore analysis of hospitalization cases may provide a good estimate of the incidence. The study assumes that the diagnosis of cCMV was based on the most current, widely used criteria. The retrospective study was performed of all patients who were hospitalized from 2009-2018. To perform statistical analyses, Statistica [24] and WINPEPI [25] were used. Means, medians, ranges for continuous variables and counts, and percentages for categorical variables were computed. Rates of cCMV-related hospitalizations were calculated as the estimated number of hospitalizations per 100,000 infants $<1$ year of age, using data from Statistics Poland [23] as the denominator. A two-sided P value less than 0.05 was considered to be statistically significant.

Formal consent of the Bioethical Commission for this type of study was not required.

\section{RESULTS}

During the study period, 2,746 hospitalization records of 1,916 patients were included in the study. The study group consisted of the 1,916 hospitalized patients ( $53 \%$ male and $47 \%$ female patients) with the first-time diagnosis of cCMV. Among them, $52 \%$ were patients up to 30 days of age. The median age was 28 days. Based on first-time hospitalizations, the average annual incidence of cCMV was 48 per 100,000 live births (95\% CI 23.5-73.4). A significantly higher cCMV incidence rate was observed in patients from more urban than rural regions of Poland (55 per 100,000 live births vs 39 per 100,000 live births, $\mathrm{P}<0.001)$. The number of first-time hospitalized cCMV cases per year are presented in Figure 1. The age in days distribution of first-time hospitalized cCMV cases is presented in Figure 2. The incidence rate of cCMV hospitalizations in Poland fluctuated during the period 20092018 (Fig. 1). Based on all hospitalizations, 9 deaths $(0.5 \%$ of all patients) were reported in the study period. Based on data from all hospitalizations, the coexistence of nervous system diseases (ICD10: G00-G99) was observed in $26 \%$ of patients, and hearing loss in $6 \%$ of patients (ICD10: H90-H91). The coexistence of hearing loss and/or neurologic diseases in the current study were $29 \%$.

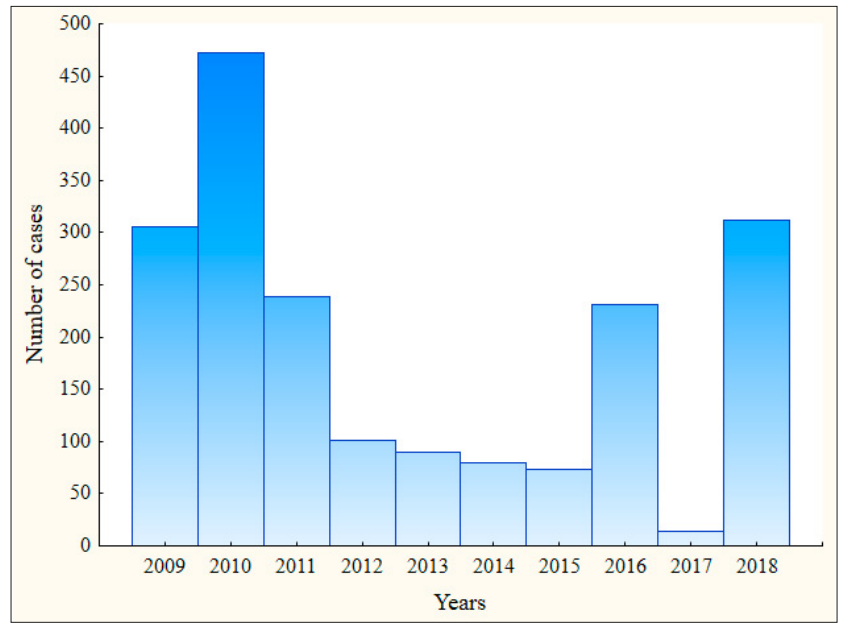

Figure 1. First-time hospitalizations of cCMV cases by year, 2009-2018

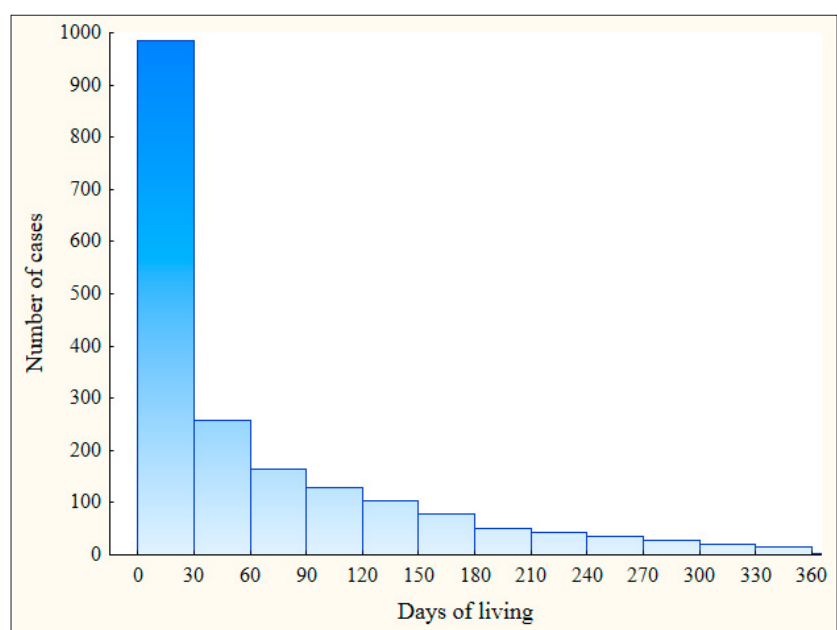

Figure 2. Age distribution of cCMV cases first-time hospitalized, 2009-2018 


\section{DISCUSSION}

Since CMV is one of the most frequent congenital infection worldwide, it is important to obtain epidemiological data on the cCMV infection as precisely as possible. Based only on the national registers of hospital morbidity, the average annual incidence of cCMV was 48 per 100,000 births in Poland. The prevalence of clinically relevant cCMV infection was between 0.008 and $0.04 \%$ in the study from Germany. [16] and 18.6 per 100,000 per year among infants $<1$ year of age in a study based on cCMV-related hospitalizations in the USA [11]; however, as reported in the other study [17], the cCMV symptoms were mostly mild. Some cases of the disease in Poland can be asymptomatic or mild and do not require hospitalization. Currently, no routine diagnostics of pregnant women for CMV infection is conducted in Poland [26]. Some cases of the disease may remain undiagnosed. In the current study, approximately 52\% of cCMV cases were observed among neonates up to 30 days of age. By comparison, data obtained from the Australian Paediatric Surveillance Unit reported that cCMV was suspected in $70.6 \%$ of patients aged under 30 days who were born between January 1999 - December 2016 [27].

The low percentage of cCMV diagnoses in this study during the first month of infants' life may also be related to the fact that cCMV tests are not routinely carried out and are probably performed only in patients with the disease symptoms. It has also been reported that the percentage of infected children with CMV-specific symptoms at birth was only $12.7 \%$ [28]. The clinical symptoms that appeared later could have been the basis for the diagnosis of cCMV. It has also been reported that at birth or in the first 2-3 weeks of life, it is essential to use appropriate tests for cCMV diagnosis, but after 2-3 weeks of life, the diagnosis of congenital infection could only be suspected on clinical grounds [29]. Moreover, in a cross-sectional study from Poland, based on data from existing serum bank from women aged $15-49$, the estimated rate of cCMV varies from 22.4-37.2 per 1,000 live births, depending on the assumptions made [18]. As presented in Figure 1, a large variability in incidence was observed over the study period. The age in days distribution of firsttime hospitalized cCMV cases is presented in Figure 2. The incidence rate of cCMV hospitalizations in Poland fluctuated over the period 2009-2018 (Fig. 1). Variability in the number of hospitalizations each year may result from both healthcare organization and the fact that $\mathrm{CCMV}$ is a rare disease in Poland. One study reported that epidemiological trends in congenital toxoplasmosis and CMV were extremely divergent. While there were only 39 cases of congenital toxoplasmosis in Switzerland between 1982-2015, there was an equivalent number of cases of cCMV - 38 in total in 2017 alone [30]. In an Australian study, hospital admissions for congenital CMV fluctuated between 1993-2001, especially for infants less than 1 month old [31]. The differences in the number of hospitalizations of newborns with CMV infections in 2009-2018 may be related to the lack of obligatory screening tests for cCMV in newborns in Poland. This problem may require separate analyzes.

The current study reports the predominance of male neonate; Statistics Poland similarly reported a slight predominance of male newborns $(51 \%)$ in relation to female newborns (49 \%) in Poland [23]. A study from the USA likewise reported that male infants with $\mathrm{CCMV}$ are more predominant [11]. In a recent study from Japan, the number of male patients was reported to be 18 out of 32 cCMV cases [32].

CCMV infection may result in damage to the developing central nervous system and may be an important cause of hearing loss. In the study group, the coexistence of nervous system diseases was observed in $26 \%$ and the hearing loss in $6 \%$ of patients. The proportion of hearing loss and/ or neurologic diseases was $29 \%$ in this study. In another study which showed that hearing loss was by far the most frequent long-term sequela in congenitall-infected infant, and occurred in $8-15 \%$ of infected infants [33]. A study conducted in France which covered data on mothers and their children aged $\geq 1$ year and diagnosed with the primary cCMV infection reported the proportion of sensorineural hearing loss and/or neurologic sequelae at the level of $32.4 \%$ [34]. In a systematic review, it was reported that $12.6 \%$ of newborns with cCMV experienced hearing loss -1 out of 3 symptomatic children and 1 out of 10 asymptomatic children [10]. Other study data from two Sydney tertiary paediatric infectious diseases clinics in Australia reported that 18 (43\%) and 17 (40\%) out of 42 infants with cCMV suffered from hearing loss and neurodevelopmental sequelae [35]. On the other hand, in Australia, human CMV was reported to be a cause of congenital malformations, affecting $\sim 0.3 \%$ of live births [36].

In the presented study it was found that there were significantly more patients living in more urban than rural regions of Poland. There may exist a relationship between the cCMV incidence and the place of residence or the socioeconomic status or other individual factors related to the newborn's mother. One study showed a correlation between cCMV occurrence and the place of birth in the south and west of America [37]. Other studies reported that CMV seroprevalence correlated with race and socioeconomic status [38]. In The Netherlands, prevalence was significantly higher among non-Western individuals $(76.7 \%)$ than the native inhabitants and those living in the west of the country (41.5\%) [39]. Data from the Pregnancy and Birth Cohort Study (20102013) conducted in Québec, Canada, showed that previous CMV infection was associated with lower education, lower income, and being born outside Canada or the United States [40]. In a study from the United States, CMV seropositivity was independently associated with low household income and low household education [41]. It was also reported that CMV infection is widespread in different age groups, and about $40-60 \%$ of adults are infected, and among people with a low socio-economic status, even above $80 \%$ [26].

In the current study, only 9 deaths ( $0.5 \%$ of all patients) were reported in the study period. In the USA, over the 17year study period from 1990-2006, 777 deaths associated with cCMV were reported, $77.7 \%$ (557 cases) of which were identified in infants aged $<1$ year [3]. In another study from the USA, approximately $4 \%$ of hospitalizations among infants $<1$ year of age resulted in death [11]. In a study from Australia, based on national mortality data sets between 1999-d 2011, childhood deaths associated with CMV occurred in 57 children under 12 months of age, with 22 cases $<1$ month of age [42].

To the best of the authors' knowledge, the current study presents for the first time selected aspects of cCMVbased on the national hospital morbidity registry, and presents recent data on the epidemiology of cCMV. The results may be helpful in comparative analyzes in the European context, 
and in taking actions aimed at improving the health of the Polish urban and rural population. Additionally, it is worth mentioning that in 1996-1998 the Institute of Physiology and Pathology of Hearing participated in the European programme 'European Concerted Action AHEAD'(Advancement of Hearing Assessment methods and Devices). The consensus on universal newborn hearing screening in Europe was signed in Italy in 1998, the signatory for Poland was Prof. Henryk Skarżyński. In January 2001, the Great Orchestra of the Christmas Charity Foundation collected funds for the implementation of a universal newborn hearing screening in Poland. The funds have been used to purchase the necessary equipment and accessories, and to train personnel in over 400 neonatology cenres. Thus, during the 10 years of the programme, more than 3,000,000 children have been screened, with the percentage of newborns in whom a congenital hearing loss was detected being $1.2 \%$ [43]. It therefore seems justified to implement in the future a programme for the early detection of CMV infection in newborns and pregnant women, and for detecting newborns at increased risk of hearing impairment or other organ complications. Such a mandatory screening test may significantly contribute to the improvement of the health of the Polish rural and urban populations.

The presented study has several limitations. Although the database used provided general information about hospitalization cases of $c \mathrm{CMV}$, thereby allowing the authors to present an epidemiological report, it did not allow them to examine risk factors and other correlates of the disease in individual patients. Using hospital discharge data limited estimation of the numbers of symptomatic cCMV-related hospitalizations for which cCMV infection was diagnosed and coded. Furthermore, the coding practices for cCMV-related hospitalizations were not assesse, and thus symptomatic cCMV-related hospitalizations may have been missed if coded using the CMV infection ICD10 code, which does not specify congenital infection. This inaccuracy may result in an underestimation of the count of incident cases. However, the large amount of data obtained from the national register of hospital morbidity for the purpose of this study may minimize the underestimation. The study did not analyze the association of cCMV with the presence of comorbidities, such as deafness or neurological diseases. Only hospital mortality was analyzed in the study, which may be lower than the overall mortality among patients with cCMV.

\section{CONCLUSIONS}

The study reported recent data on cCMV based on hospitalization data from national registers in Poland. The incidence rate of cCMV first-time hospitalizations in Poland fluctuated over the study period, with significantly more cCMV cases e observed in patients from more urban than rural regions of Poland. These observations may suggest the presence of territorial or other factors related to the cCMV incidence in Poland. The relatively high percentage of newborns with hearing loss or neurologic diseases may require activities aimed at reducing the cCMV incidence. The epidemiological data presented may be useful for comparisons with other geographical regions.

\section{REFERENCES}

1. Rawlinson WD, Boppana SB, Fowler KB, et al. Congenital cytomegalovirus infection in pregnancy and the neonate: consensus recommendations for prevention, diagnosis, and therapy. Lancet Infect Dis. 2017; 17(6): e177-188. https://doi.org/10.1016/S1473-3099(17)30143-3

2. Manicklal S, Emery VC, Lazzarotto T, et al. The "silent" global burden of congenital cytomegalovirus. Clin Microbiol Rev. 2013; 26(1): 86-102. https://doi.org/10.1128/CMR.00062-12

3. Bristow BN, O'Keefe KA, Shafir SC, et al. Congenital cytomegalovirus mortality in the United States, 1990-2006. PLoS Negl Trop Dis. 2011; 5(4): e1140. https://doi.org/10.1371/journal.pntd.0001140

4. Pereira L. Congenital Viral Infection: Traversing the Uterine-Placental Interface. Annu Rev Virol. 2018 29; 5(1): 273-99. https://doi.org/10.1146/ annurev-virology-092917-043236

5. Picone O, Vauloup-Fellous C, Cordier AG, et al. A series of 238 cytomegalovirus primary infections during pregnancy: description and outcome. Prenat Diagn. 2013; 33(8): 751-758. https://doi.org/10.1002/ pd.4118

6. Kobas M, Bickle Graz M, Truttmann AC, et al. Clinical characteristics, audiological and neurodevelopmental outcomes of newborns with congenital cytomegalovirus infection. Swiss Med Wkly. 2018; 148: w14627. https://doi.org/10.4414/smw.2018.14627

7. Leruez-Ville M, Magny J-F, Couderc S, et al. Risk Factors for Congenital Cytomegalovirus Infection Following Primary and Nonprimary Maternal Infection: A Prospective Neonatal Screening Study Using Polymerase Chain Reaction in Saliva. Clin Infect Dis. 2017; 65(3): 398-404. https://doi.org/10.1093/cid/cix337

8. Leruez-Ville M, Guilleminot T, Stirnemann J, et al. Quantifying the burden of congenital CMV infection (cCMV) with long-term sequelae in subsequent pregnancies of women seronegative at their first pregnancy. Clin Infect Dis, ciz1067. https://doi.org/10.1093/cid/ciz1067

9. Mestas E. Congenital Cytomegalovirus. Adv Neonatal Care. 2016; 16(1): 60-65. https://doi.org/10.1097/ANC.0000000000000242

10. Goderis J, De Leenheer E, Smets K, et al. Hearing loss and congenital CMV infection: a systematic review. Pediatrics. 2014; 134(5): 972-982. https://doi.org/10.1542/peds.2014-1173

11. Lopez AS, Ortega-Sanchez IR, Bialek SR. Congenital cytomegalovirusrelated hospitalizations in infants $<1$ year of age, United States, 1997 2009. Pediatr Infect Dis J. 2014; 33(11): 1119-23. https://doi.org/10.1097/ INF.0000000000000421

12. Leung J, Kennedy JL, Haberling DL, et al. Congenital CMV-Coded Diagnosis Among American Indian and Alaska Native Infants in the United States, 2000-2017. J Immigr Minor Health. 2020; 22(5): 1101-1104. https://doi.org/10.1007/s10903-020-01024-3

13. Lanzieri TM, Dollard SC, Bialek SR, et al. Systematic review of the birth prevalence of congenital cytomegalovirus infection in developing countries. Int J Infect Dis. 2014; 22: 44-48. https://doi.org/10.1016/j. ijid.2013.12.010

14. Putri ND, Wiyatno A, Dhenni R, et al. Birth prevalence and characteristics of congenital cytomegalovirus infection in an urban birth cohort, Jakarta, Indonesia. Int J Infect Dis. 2019; 86: 31-39. https:// doi.org/10.1016/j.ijid.2019.06.009

15. Wang S, Wang T, Zhang W, et al. Cohort study on maternal cytomegalovirus seroprevalence and prevalence and clinical manifestations of congenital infection in China. Medicine (Baltimore). 2017; 96(5): e6007. https://doi.org/10.1097/MD.0000000000006007

16. Rütten H, Rissmann A, Brett B, et al. Congenital cytomegalovirus infection in Central Germany: an underestimated risk. Arch Gynecol Obstet. 2017; 296(2): 231-240. https://doi.org/10.1007/s00404-0174435-4

17. Townsend CL, Forsgren M, Ahlfors K, et al. Long-term outcomes of congenital cytomegalovirus infection in Sweden and the United Kingdom. Clin Infect Dis. 2013; 56(9): 1232-1239. https://doi. org/10.1093/cid/cit018

18. Siennicka J, Dunal-SzcepaniakM, Trzcińska A, et al. High Seroprevalence of CMV Among Women of Childbearing Age Implicates High Burden of Congenital Cytomegalovirus Infection in Poland. Pol J Microbiol. 2017; 65(4): 425-432. https://doi.org/10.5604/17331331.1227668

19. Rycel M, Wujcicka W, Zawilińska B, et al. Mixed infections with distinct cytomegalovirus glycoprotein B genotypes in Polish pregnant women, fetuses, and newborns. Eur J Clin Microbiol Infect Dis. 2015; 34(3): 585-591. https://doi.org/10.1007/s10096-014-2266-9

20. Paradowska E, Jabłońska A, Studzińska M, et al. Distribution of the CMV glycoprotein $\mathrm{gH} / \mathrm{gL} / \mathrm{gO}$ and $\mathrm{gH} / \mathrm{gL} / \mathrm{pUL128} / \mathrm{pUL} 130 / \mathrm{pUL131A}$ complex variants and associated clinical manifestations in infants 
infected congenitally or postnatally. Sci Rep. 2019 08; 9(1): 16352. https:// doi.org/10.1038/s41598-019-52906-y

21. Kasztelewicz B, Czech-Kowalska J, Lipka B, et al. Cytokine gene polymorphism associations with congenital cytomegalovirus infection and sensorineural hearing loss. Eur J Clin Microbiol Infect Dis. 2017; 36(10): 1811-1818. https://doi.org/10.1007/s10096-017-2996-6

22. Sobolewska-Pilarczyk M, Pawlak-Osinska K, Drewa S, et al. Intraventricular haemorrhage as the first manifestation of congenital Cytomegalovirus infection. Indian J Med Microbiol. 2018; 36(2): 279-281. https://doi.org/10.4103/ijmm.IJMM_18_11

23. Statistics Poland. http://stat.gov.pl. (access: 2020.03.07)

24. TIBCO Software Inc. (2017). Statistica (data analysis software system), version 13. http://statistica.io.

25. Abramson JH. WINPEPI updated: computer programs for epidemiologists, and their teaching potential. Epidemiol Perspect Innov. 2011; 8: 1. https://doi.org/10.1186/1742-5573-8-1

26. Pokorska-Śpiewak M, Niezgoda A, Gołkowska M, et al. Recommendations for the diagnosis and treatment of CMV infections. Polish Society of Epidemiology and Infectious Diseases. Przegl Epidemiol. 2016; 70(2): 297-310.

27. Bartlett AW, Hall BM, Palasanthiran P, et al. Recognition, treatment, and sequelae of congenital cytomegalovirus in Australia: An observational study. J Clin Virol. 2018; 108: 121-125. https://doi. org/10.1016/j.jcv.2018.09.017

28. Dollard SC, Grosse SD, Ross DS. New estimates of the prevalence of neurological and sensory sequelae and mortality associated with congenital cytomegalovirus infection. Rev Med Virol. 2007; 17(5): 355-363. https://doi.org/10.1002/rmv.544

29. Lazzarotto T, Guerra B, Lanari M, et al. New advances in the diagnosis of congenital cytomegalovirus infection. J Clin Virol. 2008; 41(3): 192-197. https://doi.org/10.1016/j.jcv.2007.10.015

30. L'Huillier AG, Crisinel PA. Toxoplasmose et cytomégalovirus congénitaux en Suisse en 2019 [Congenital Toxoplasmosis and CMV in Switzerland in 2019]. Rev Med Suisse. 2020; 16(682): 361-364. [Abstract in English, French]. https://pubmed.ncbi.nlm.nih.gov/32073771/ (access: 2020.09.27)

31. Seale H, Booy R, MacIntyre CR. Trends in hospitalizations for diagnosed congenital cytomegalovirus in infants and children in Australia. BMC Pediatr 2009; 9: 1. https://doi.org/10.1186/1471-2431-9-7

32. Ohyama S, Fujioka K, Fukushima S, et al. Diagnostic Value of Cytomegalovirus IgM Antibodies at Birth in PCR-Confirmed Congenital Cytomegalovirus Infection. Int J Mol Sci. 2019; 20(13): 3239. https:// doi.org/10.3390/ijms20133239
33. Fowler KB. Congenital cytomegalovirus infection: audiologic outcome. Clin Infect Dis. 2013; 57 Suppl 4: S182-184. https://doi.org/10.1093/ cid/cit609/

34. Faure-Bardon V, Magny J-F, Parodi M, et al. Sequelae of Congenital Cytomegalovirus Following Maternal Primary Infections Are Limited to Those Acquired in the First Trimester of Pregnancy. Clin Infect Dis. 2019; 69(9): 1526-1532. https://doi.org/10.1093/cid/ciy1128

35. McMullan BJ, Palasanthiran P, Jones CA, et al. Congenital cytomegalovirus - time to diagnosis, management and clinical sequelae in Australia: opportunities for earlier identification. Medical Journal of Australia. 2011; 194(12): 625-629. https://doi. org/10.5694/j.1326-5377.2011.tb03144.x

36. Naing ZW, Scott GM, Shand A, et al. Congenital cytomegalovirus infection in pregnancy: a review of prevalence, clinical features, diagnosis and prevention. Aust N Z J Obstet Gynaecol. 2016; 56(1): 9-18. https://doi.org/10.1111/ajo.12408

37. Inagaki K, Blackshear C, Palmer A, et al. Risk Factors, Geographic Distribution, and Healthcare Burden of Symptomatic Congenital Cytomegalovirus Infection in the United States: Analysis of a Nationally Representative Database, 2000-2012. J Pediatr. 2018; 199: 118-123.e1. https://doi.org/10.1016/j.jpeds.2018.03.036

38. Cannon MJ, Schmid DS, Hyde TB. Review of cytomegalovirus seroprevalence and demographic characteristics associated with infection. Rev Med Virol. 2010; 20(4): 202-213. https://doi.org/10.1002/ rmv. 655

39. Korndewal MJ, Mollema L, Tcherniaeva I, et al. Cytomegalovirus infection in the Netherlands: seroprevalence, risk factors, and implications. J Clin Virol. 2015; 63: 53-58. https://doi.org/10.1016/j. jcv.2014.11.033

40. Lamarre V, Gilbert NL, Rousseau C, et al. Seroconversion for cytomegalovirus infection in a cohort of pregnant women in Québec, 2010-2013. Epidemiol Infect. 2016; 144(8): 1701-1709. https://doi. org/10.1017/S0950268815003167

41. Bate SL, Dollard SC, Cannon MJ. Cytomegalovirus seroprevalence in the United States: the national health and nutrition examination surveys, 1988-2004. Clin Infect Dis. 2010; 50(11): 1439-1447. https:// doi.org $/ 10.1086 / 652438$

42. Smithers-Sheedy H, Raynes-Greenow C, Badawi N, et al. Cytomegalovirus-related childhood mortality in Australia 1999-2011. J Paediatr Child Health. 2015; 51(9): 901-905. https://doi.org/10.1111/jpc.12896

43. Universal Hearing Screening Programs. https://whc.ifps.org. $\mathrm{pl} / \mathrm{en} / 2011 / 12 /$ universal-hearing-screening-programs/. (access: 2020.09.27) 